\title{
Videojuego móvil como estrategia didáctica para facilitar la adaptación a la vida universitaria
}

\author{
Julian Moreno* y Juan A. Álvarez \\ Departamento de Ciencias de la Computación y la Decisión, Universidad Nacional de Colombia Sede Medellín, Carrera \\ 80 65-223, Medellín - Colombia. (e-mail: jmoreno1@unal.edu.co, juaalvarezme@unal.edu.co) \\ * Autor a quien debe ser dirigida la correspondencia
}

Recibido Abr. 18, 2019; Aceptado Jun. 24, 2019; Versión final Ago. 29, 2019, Publicado Feb. 2020

\begin{abstract}
Resumen
Se describe un videojuego para apoyar el proceso de inducción a estudiantes universitarios teniendo como caso de aplicación específico la Universidad Nacional de Colombia. Para su diseño se adoptó la metodología 6i para juegos serios y dentro de su componente instruccional se consideraron cuatro aspectos que son recurrentes en la literatura como claves para el proceso de transición desde la educación básica. Estos son, los hábitos de estudio, los hábitos de vida saludable, el entendimiento de varios conceptos académicos, y el reconocimiento del campus. Junto con su diseño, se presenta también una validación realizada mediante un estudio de caso con 75 estudiantes provenientes de 20 programas universitarios. Dicha validación consideró la evaluación de la percepción mediante una encuesta tipo Likert. Se encontró que la mayoría de los encuestados estuvieron de acuerdo con las diferentes características relacionadas con la jugabildad, pero principalmente, que lo encontraron útil para interiorizar los cuatro aspectos mencionados.
\end{abstract}

Palabras clave: juegos serios; modelo 6i; aprendizaje móvil; hábitos de estudio; hábitos de vida saludable

\section{Mobile videogame as a didactic strategy to facilitate college adjustment}

\begin{abstract}
A videogame to support the induction process to college students and having as specific application case the National University of Colombia is described. For its design, the 6i methodology for serious games was followed and, within its instructional component, four aspects that are recurrent in the literature as key for the transition from basic education were considered. These are, study habits, healthy life habits, the understanding of various academic concepts, and the campus recognition. Along with its design, a validation is also presented through a case study with 75 students from 20 university programs. Such validation considered the perception evaluation by means of a Likert survey. It was found that the majority of the students agreed with the different characteristics related to the gameplay, but mainly, that they found it useful to internalize the four above mentioned aspects.
\end{abstract}

Keywords: serious games; 6i model; m-learning; study habits; healthy life habits 


\section{INTRODUCCIÓN}

El paso de un estudiante de la enseñanza media a la educación superior suele ser un proceso difícil. Para autores como Díaz (2008), las dificultades comienzan desde que los estudiantes son obligados a transitar de un ambiente conocido y relativamente seguro como es la escuela, a un mundo en apariencia impersonal como la universidad donde deben valerse por sí mismos, no solo en las aulas de clase, sino en general en los diferentes espacios institucionales. Más aún, tal como lo señalan Wang et al. (2006), en muchos de los casos dicho proceso coincide con un punto de inflexión de la vida de los estudiantes en el que pasan de la adolescencia a la adultez temprana. Todo esto hace que el ingreso a la vida universitaria se constituya en un reto, en el cual si los estudiantes no se adaptan de forma efectiva puede significar problemas importantes no solo en su desarrollo académico sino también psicológico.

Tan es así en el caso académico que, pese a que diversas investigaciones sitúan la situación económica y el rendimiento académico como dos de las principales causas de la deserción universitaria, algunas también suelen incluir en el listado a la adaptación inadecuada como una de ellas (Wilson, 1984; Corominas, 2001; Cabrera et al., 2006; Taylor y Pastor, 2007; Castaño et al., 2008; Wintre et al., 2008; Díaz, 2009; Etxeberria et al., 2017; Miranda y Guzmán, 2017; Gallegos et al., 2018; Munizaga et al., 2018). De igual manera, otras investigaciones buscan analizar de forma detallada tanto los problemas asociados a dicha adaptación como sus posibles soluciones. Entre estas, se destacan aquellas que ponen en manifiesto la importancia de factores sociales como es el caso de la de Figuera et al. (2003), Wang et al. (2006) y Julia y Veni (2012) donde se analiza el apoyo familiar o de amigos dentro del campus y su influencia en las expectativas de logro universitario; así como la de Abe et al. (1998) y Mattanah et al. (2010) donde se examina el efecto de los grupos de soporte conformados por pares, y la de Arjanggi y Kusumaningsih (2016) donde se estudia en rol de la ansiedad social durante el proceso de ajuste. Otras se centran en factores personales, como es el caso de Parker et al. (2004) donde examinan la relación entre la inteligencia emocional y el desempeño académico, o la de Pancer et al. (2000) donde evalúan la incidencia de la complejidad de expectativas de los estudiantes al ingreso a la universidad frente a su desenvolvimiento en circunstancias de alto estrés.

Muchos de los trabajos previamente citados se fundamentan en modelos de orden sociológicos tanto teóricos como empíricos que buscan relacionar las diversas causales de la deserción. Entre ellos, se destacan los desarrollados por Spady (1971), Tinto (1975) y Bean (1980). El primero afirma que la deserción es el resultado de un proceso social complejo que incluye factores tan diversos como los antecedentes familiares, la educación previa, el potencial académico, la congruencia normativa, el apoyo de amigos, el desarrollo intelectual, la integración social, la satisfacción y el compromiso institucional. El segundo por su parte argumenta que las causas, más que originarse de habilidades inadecuadas de los estudiantes, se originan de una integración personal insuficiente con los ambientes intelectual y social de la comunidad institucional. Entre tanto el tercero, más que en factores institucionales, se concentra en aquellos intrínsecos al estudiante, y en especial en los parámetros psicológicos que afectan su intención de continuar o abandonar sus estudios.

Teniendo en cuenta este panorama, muchas universidades brindan procesos de inducción para los recién admitidos que incluyen pautas para llevar a cabo tal adaptación. Esto suele hacerse bien sea en el periodo entre la admisión y el inicio de clases, o bien durante el primer periodo académico. Su intensidad y formato también pueden variar, puede tratarse de jornadas de uno o pocos días, pasando por cursos cortos de algunas semanas, o incluso cursos completos de la misma duración que los cursos regulares. Dicha aproximación sin embargo no siempre resulta efectiva, bien sea por la didáctica con que se lleva a cabo, o porque los contenidos son insuficientes, o simplemente porque los estudiantes no le dan la suficiente importancia. En su investigación sobre los comportamientos de estudiantes recién admitidos en una Universidad Colombiana, Cardona et al. (2011) encontraron que, pese a existir un proceso de inducción estructurado, para muchos no era del todo adecuado pues a su sentir los contenidos eran demasiado generales y no apuntaban a las necesidades particulares de quienes están ingresando a la universidad. De igual manera, encontraron además que en términos generales el proceso en sí les era poco interesante y de hecho no era identificado por los estudiantes como un acto de acogida sino como un mero proceso administrativo.

Como una alternativa para resolver esta problemática, en este artículo se propone un modelo de inducción con dos características diferenciadoras. La primera es que incluye de manera integrada tres de los puntos claves referenciados por las investigaciones previamente citadas sobre la adaptación a la vida universitaria: los hábitos de estudio, los hábitos de vida saludable, y el entendimiento de ciertos conceptos que suelen ser confusos para los estudiantes. La segunda, y siendo un aspecto innovador del modelo en cuanto a la didáctica de implementación, es que se hace mediante un formato que suele ser muy familiar y atrayente para la población objetivo, un videojuego. Con esto último se busca romper con el esquema tradicional que como se mencionó suele no resultar atractivo para la población objetivo. Siendo así, lo que se describe en el resto del documento es el diseño de un video juego para la inducción a la vida universitaria, usando caso particular la Universidad Nacional de Colombia Sede Medellín. De forma más específica, se trata de un juego serio, 
traducción de serious game, termino se refiere a un juego que no tiene un fin netamente lúdico sino educativo. El mismo cuenta además con otra particularidad y es que se implementó como una aplicación móvil, buscando brindar una mayor flexibilidad de uso. Posterior a la descripción de su diseño, se presenta también una validación de corte cuantitativo mediante una encuesta de percepción usando una escala tipo Likert.

\section{DISEÑO DEL VIDEOJUEGO}

La clave del éxito de un juego serio es la identificación de los aspectos principales que permiten un balance mediante el cual el jugador lo perciba como una actividad divertida pero cuyo objetivo real sea propiciar una experiencia de aprendizaje. Para lograr esto, en esta propuesta se siguió la metodología de las seis 'i' (6I) para el diseño de juegos serios (Annetta, 2006), denominada así pues considera los seis aspectos que se presentan en la figura 1 , los cuales aparecen en orden de magnitud y de manera anidada.

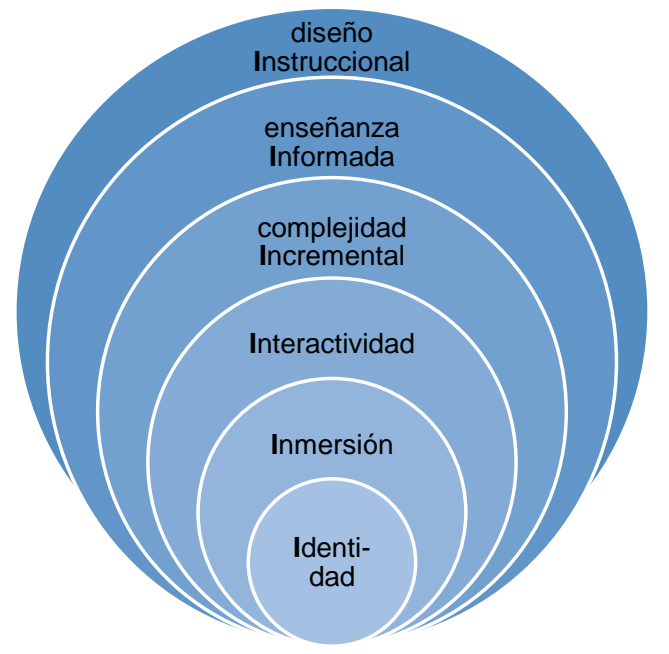

Fig. 1: Aspectos del modelo 6I (Adaptada de Annetta, 2010)

La Identidad (I1) es la capacidad de representar al jugador de manera que sienta que es él o ella quien se encuentra dentro del juego. En muchos juegos esto se logra por medio de un carácter único llamado avatar. La Inmersión (I2) se logra cuando el jugador tiene un alto sentido de presencia dentro del juego, y se integra a sí mismo en su narrativa como responsable de alcanzar el éxito del reto que esta le impone. La Interactividad (I3) hace referencia a la interconexión de las diferentes partes del juego entre sí, con el jugador, y entre los jugadores. Debe existir una realimentación continua y el jugador debe poder percibir las consecuencias de sus acciones. La complejidad Incremental (14) busca mantener la atención del jugador para que en la medida que avance dentro del juego, la dificultad de los desafíos que se le presentan también lo haga. La enseñanza Informada (15) significa, no solo que el juego debe incorporar uno o más mecanismo de puntaje para representar el progreso del jugador, sino que las mecánicas de juego de desencadenan tales puntajes deben, en la medida de lo posible, reflejar el nivel de aprendizaje adquirido. El diseño Instruccional (16) es donde se establece que el juego puede ser usado como un medio de enseñanza. En este se debe establecer cuál es el objetivo de aprendizaje (qué se va a aprender) y debe permear los otros cinco aspectos.

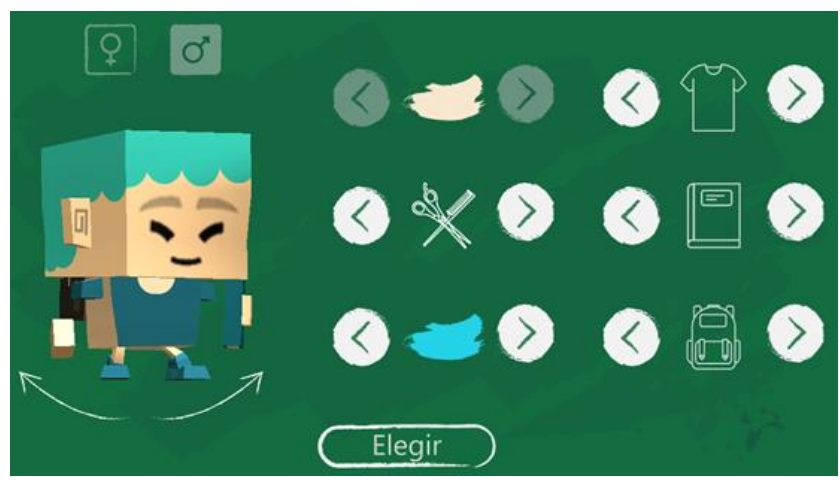

Fig. 2: Interfaz de personalización del avatar.

En nuestra propuesta estos seis aspectos fueron considerados, aunque no en el mismo orden, de esta manera: El juego comienza con un intro, o historia de presentación, en el cual un personaje con apariencia de rector o decano le informa al jugador que acaba de ser admitido a la Universidad. Lo felicita por este logro, 
pero le advierte que es solo el primer paso de un enorme reto que es terminar de forma exitosa una carrera universitaria (I2: Inmersión). Acto seguido el jugador debe personalizar su avatar antes de comenzar el primer semestre (I1: Identidad), tal como se muestra en la figura 2. Siguiendo con su charla, el personaje le muestra el campus de la Universidad, lugar en el cual transcurrirá el juego (I2: Inmersión). En este caso corresponde al campus de la Universidad Nacional de Colombia Sede Medellín. A partir de ese momento el jugador puede comenzar a recorrerlo y a interactuar con otros personajes y elementos dispersos por todo el campus como se muestra en la figura 3 (I3: Interactividad).

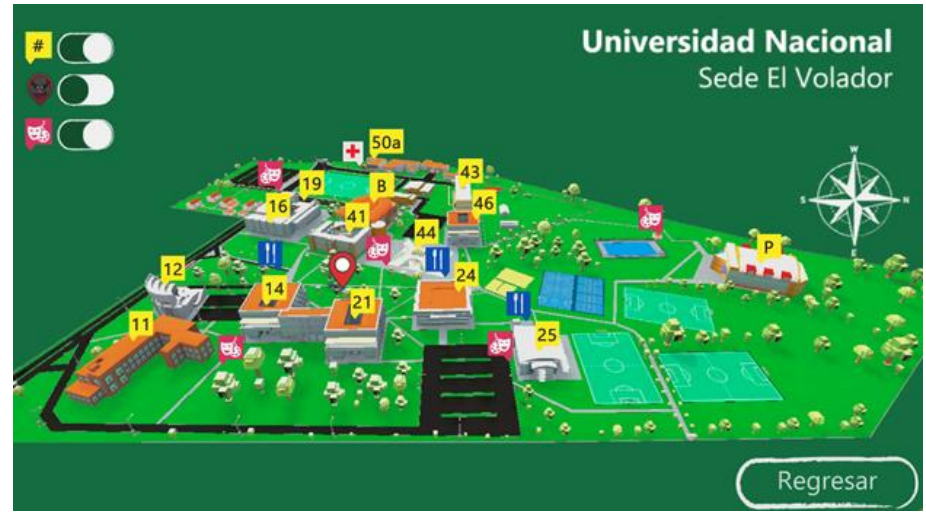

Fig. 3: Mundo virtual que representa el campus Universitario

La idea del juego es brindar una representación animada de la vida universitaria: los niveles corresponden a diferentes semestres que el estudiante va cursando (I4: complejidad Incremental). Cada semestre tiene un calendario académico que es visible para el jugador como se muestra en la figura 4, y el reto del juego se va traduciendo en "ganar" cada uno de ellos. A la hora del diseño Instruccional (I6) se buscó que el estudiante aprendiera sobre tres temas específicos: buenos hábitos de estudio, hábitos de vida saludable, y algunos conceptos académicos. Un cuarto tema, menos significativo pero igualmente relevante en muchos procesos de inducción, es el reconocimiento del campus (Vendrell y Sanchez, 2011), que en el caso del juego está íntimamente ligado a la Inmersión (I2).
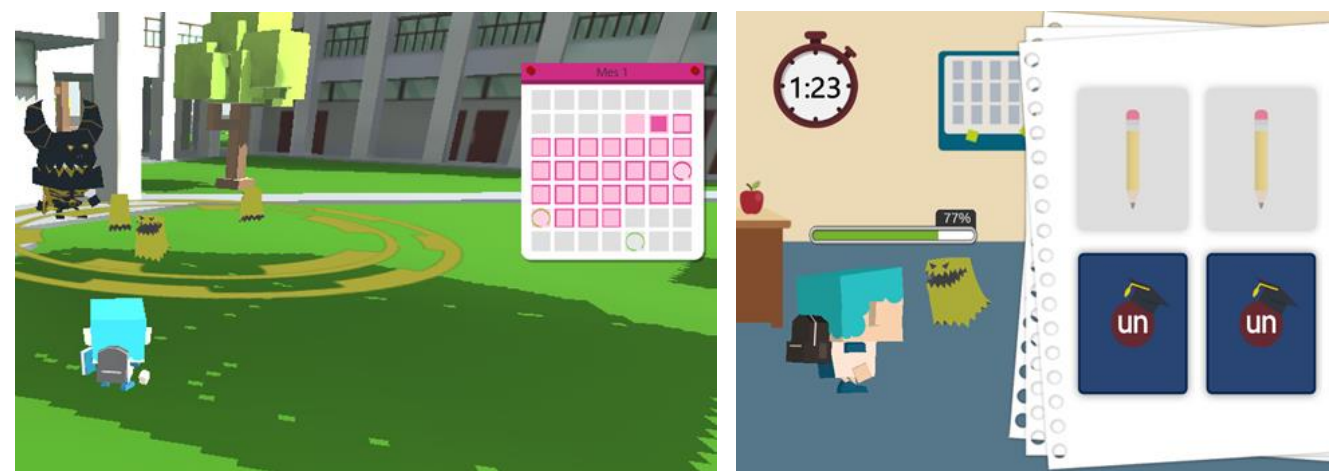

Fig. 4: Interfaces de enfrentamientos

Con respecto a los buenos hábitos de estudio (Credé y Kuncel, 2008; Yu, 2011), la mecánica del juego establece que el jugador puede enfrentar en cualquier momento a contendientes menores, representados como se muestra en la parte izquierda de la figura 4 como pequeños fantasmas, que corresponden dentro de la metáfora del juego a las lecturas, repasos, tareas, talleres, u otros medios de estudio. Lo que no puede enfrentar en el momento que desee, sino cuando el calendario académico lo establezca, son los contendientes mayores representados por monstruos de mayor tamaño y que corresponden dentro de la metáfora a las evaluaciones de los cursos como exámenes, trabajos u otros. Los enfrentamientos se corresponden a minijuegos como se muestra en la parte derecha de la misma figura y la idea es que en la medida que el jugador venza a los fantasmas adquirirá más puntos de experiencia o XP se expresa comúnmente en la terminología de juegos. Entre más XP se tengan más factible será vencer a los monstruos y viceversa. Siendo así, lo que se espera que el estudiante interiorice es que el estudio es un proceso permanente y que un buen hábito de estudio es realizar esas actividades de aprendizaje de forma constante, no solo en víspera de las evaluaciones. Esta mecánica de juego incorpora además el aspecto de la complejidad Incremental (14) pues la dificultad para ganarle a los contendientes aumenta a medida que avanzan los semestres.

Con respecto a los hábitos de vida saludable (Abe et al., 1998), parte de la mecánica del juego establece además que, en la medida que el jugador lleva a cabo sus labores diarias de estudio y desplazamiento por el campus, dos procesos "negativos" le suceden a su avatar: disminuye su nivel de energía y aumenta el de 
estrés. Para que dichos niveles no lleguen a niveles peligrosos, caso en el cual el juego se pierde, es necesario que el estudiante aprenda la importancia de una buena alimentación y de llevar a cabo actividades deportivas y lúdicas. En este caso esta metáfora se establece cuando el jugador "toma" alguno de los menús saludables disponibles en las caferías dispersas por el campus como se muestra en la parte izquierda de la figura 5. Así mismo, y para disminuir el estrés, el jugador puede interactuar con otros personajes también dispersos por el campus, para realizar acciones como relajarse en la piscina, hacer ejercicio, escuchar música, entre otras, tal como se muestra en la parte derecha de la misma figura.
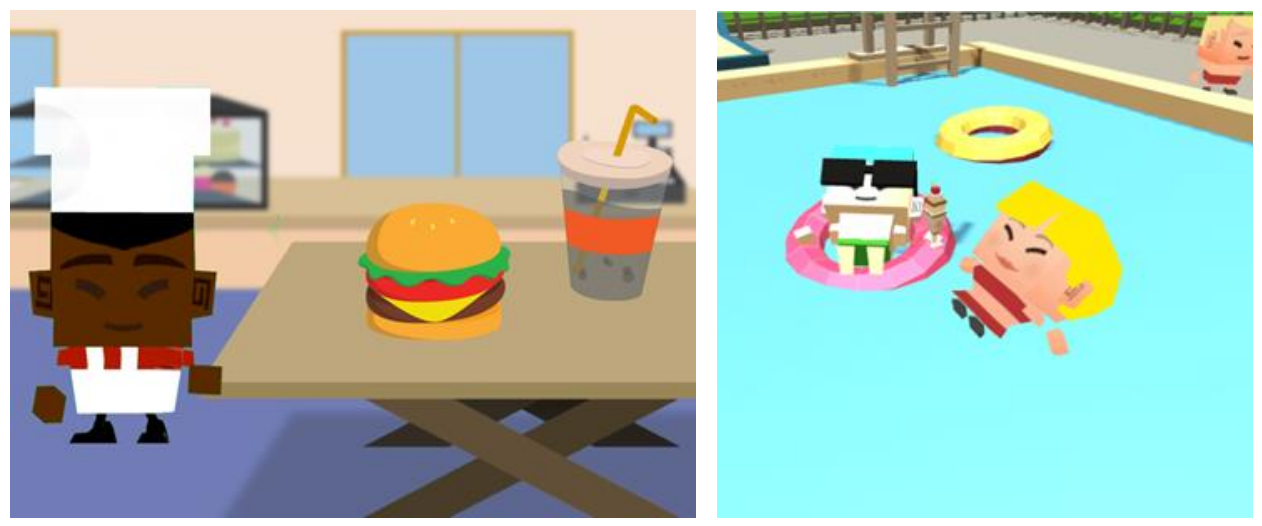

Fig. 5: Interfaces de interacción en la cafetería (izquierda) y piscina (derecha) del campus

Respecto a los conceptos académicos, se consideraron cuatro que pueden resultar confusos para muchos de los estudiantes que recién llegan de la enseñanza media: porcentaje (peso) de una evaluación, nota acumulada, crédito, y promedio académico ponderado. Como se muestra en la figura 6 , cada semestre se compone de un conjunto de cursos genéricos. Cada curso tiene asociado una cantidad de créditos y tiene una serie de evaluaciones.

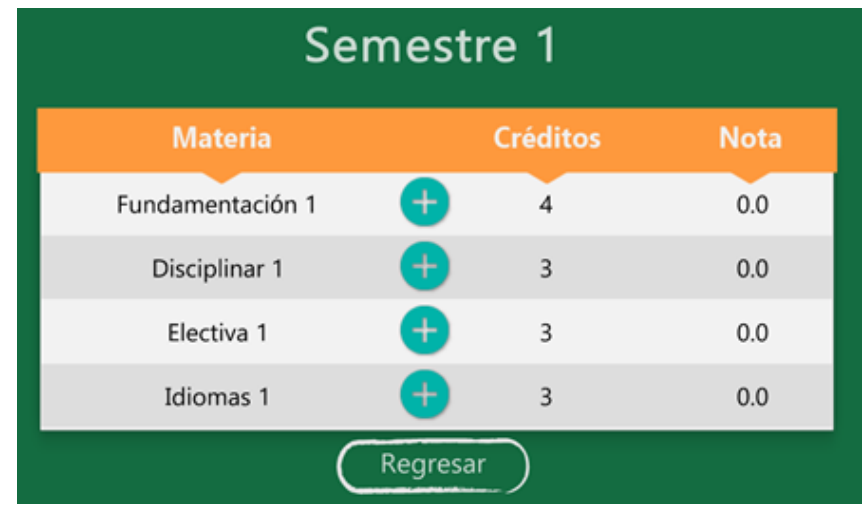

Fig. 6: Interfaz de presentación de notas acumuladas de semestre

Cuando un estudiante dentro del juego realiza una evaluación, es decir, se enfrenta a un monstruo, se le asigna una calificación entre 0 y 5 con una cifra decimal. Dicha calificación dependerá de que tan bien o mal le vaya en el enfrentamiento, lo cual como ya se explicó dependerá a su vez de que tanto haya "estudiado antes", es decir, enfrentado previamente fantasmas. Al obtener dicha calificación, es estudiante recibe como realimentación adicional una animación con el cálculo de la nota acumulada, que corresponde a la sumatoria de las evaluaciones realizadas, multiplicadas por su porcentaje correspondiente y dividido por el porcentaje evaluado acumulado. De forma similar, pero al terminar cada semestre, se realimenta al estudiante con una animación del cálculo del promedio académico ponderado para lo cual se usan las calificaciones finales de todos los cursos finalizados, ponderándolos con los créditos correspondientes.

\section{METODOLOGÍA DE VALIDACIÓN Y RESULTADOS}

Para validar la propuesta se llevó a cabo un estudio de caso con estudiantes recién admitidos de la Universidad Nacional de Colombia Sede Medellín. El videojuego se montó en la tienda de aplicaciones de google play, bajo el nombre IntroUN y se compartió el enlace para que aquellos que de manera voluntaria quisieran usarlo pudieran hacerlo. De igual manera se publicó una encuesta para que a aquellos que lo descargaran y jugaran, respondieran de forma libre. Tal encuesta consistió de diez preguntas a manera de afirmación para que los estudiantes respondieran con una escala tipo Likert con las siguientes opciones: 1: Muy en desacuerdo; 2: En desacuerdo, 3: No estoy seguro(a); 4: De acuerdo; y 5: Muy de acuerdo. Tales 
preguntas se presentan en la tabla 1, las seis primeras orientadas a la percepción sobre la jugabilidad y las cuatro restantes orientadas a la percepción sobre la efectividad en el aprendizaje.

Tabla 1: Encuesta de percepción

\begin{tabular}{|c|l|}
\hline Pregunta & Formulación \\
\hline P1 & La interfaz del juego y su estética en general me pareció agradable \\
\hline P2 & Me gustó la personalización del avatar \\
\hline P3 & Los iconos y botones son claros y fáciles de usar \\
\hline P4 & La navegación dentro del juego es simple y consistente \\
\hline P5 & Las realimentaciones brindadas son claras y pertinentes \\
\hline P6 & Que el juego fuera para móvil facilitó su uso \\
\hline P7 & Mejoré mi conocimiento sobre el campus de la Universidad, la ubicación de los edificios y lo que albergan \\
\hline P8 & $\begin{array}{l}\text { Entendí claramente la metáfora sobre importancia de manejar bien el tiempo, estudiar y prepararse para } \\
\text { las evaluaciones }\end{array}$ \\
\hline P9 & $\begin{array}{l}\text { Entendí claramente la metáfora de alimentarse bien y de complementar el estudio con actividades } \\
\text { deportivas y lúdicas }\end{array}$ \\
\hline P10 & Mejoré mi comprensión sobre el cálculo de nota acumulada y promedio ponderado \\
\hline
\end{tabular}

Luego de un periodo de cuatro semanas, un total de 75 estudiantes jugaron y respondieron la encuesta. Este valor equivale aproximadamente al $7 \%$ de la población objetivo que son cerca de 1200 admitidos por semestre. La moda de la edad fue de 18 años, siendo el promedio 18.46 y la desviación estándar 1.81. Como se observa en la tabla 2, hubo una participación de 20 de los 27 programas de pregrado ofertados por la Universidad, siendo la participación por programa relativamente coherente con la cantidad de admitidos de cada uno. Algo similar ocurre con el porcentaje entre hombres y mujeres.

Tabla 2: Porcentaje de participación por programa de pregrado y género

\begin{tabular}{|l|c|c|c|l|c|c|c|}
\hline \multicolumn{1}{|c|}{ Carrera } & Hombres & Mujeres & Total & Carrera & Hombres & Mujeres & Total \\
\hline Ingeniería Eléctrica & 8 & 4 & 12 & Ingeniería Mecánica & 2 & 1 & 3 \\
\hline $\begin{array}{l}\text { Ingeniería de Sistemas e } \\
\text { Informática }\end{array}$ & 7 & 2 & 9 & Historia & 0 & 2 & 2 \\
\hline Economía & 6 & 2 & 8 & Ingeniería Agronómica & 2 & 0 & 2 \\
\hline Ingeniería Civil & 5 & 3 & 8 & Ingeniería de Petróleos & 2 & 0 & 2 \\
\hline Estadística & 1 & 5 & 6 & Ciencia Política & 1 & 0 & 1 \\
\hline Ingeniería Ambiental & 2 & 2 & 4 & Construcción & 1 & 0 & 1 \\
\hline Ingeniería Industrial & 2 & 2 & 4 & Ingeniería de Control & 1 & 0 & 1 \\
\hline Arquitectura & 2 & 1 & 3 & Ingeniería Geológica & 1 & 0 & 1 \\
\hline Ingeniería Física & 2 & 1 & 3 & Ingeniería Química & 1 & 0 & 1 \\
\hline Ingeniería Forestal & 0 & 3 & 3 & Matemáticas & 1 & 0 & 1 \\
\hline
\end{tabular}

El total de las respuestas obtenidas en la encuesta se presentan en la figura 7. Adicionalmente, y considerando que todas las respuestas se encuentran en la misma escala Likert, el agregado de las relacionadas a la jugabilidad (preguntas 1 a 6) y a la efectividad de aprendizaje (preguntas 7 a 10) se presenta en la figura 8.

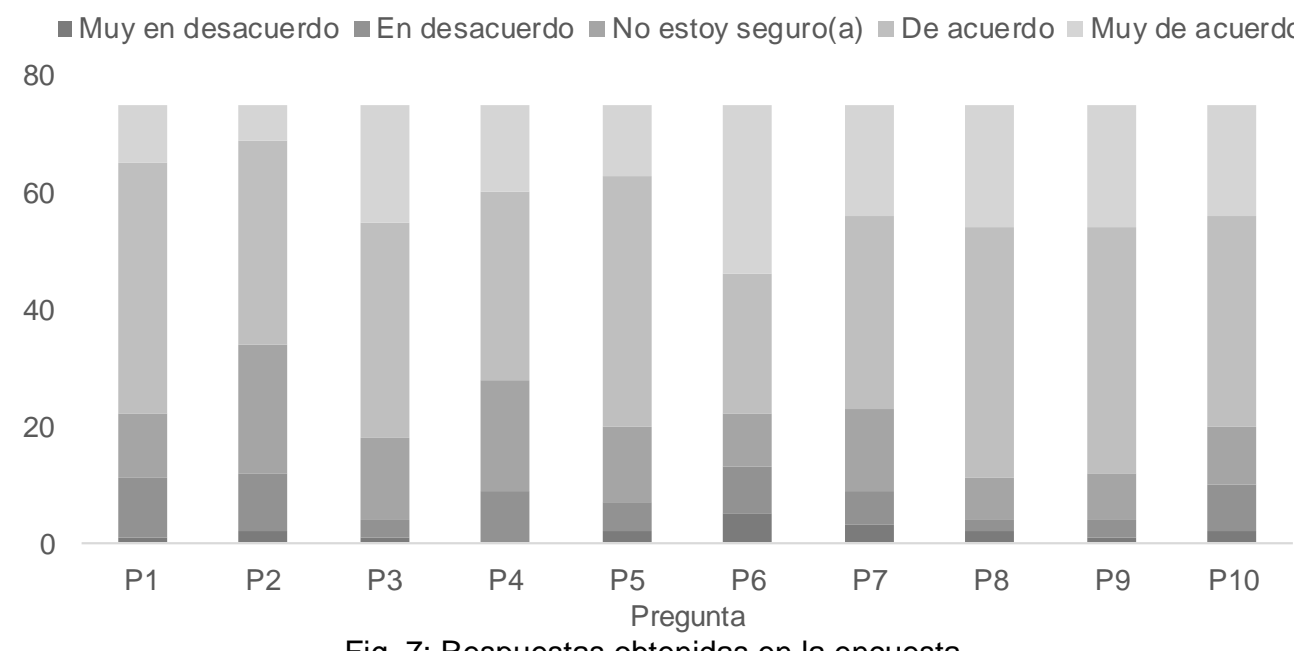

Fig. 7: Respuestas obtenidas en la encuesta 


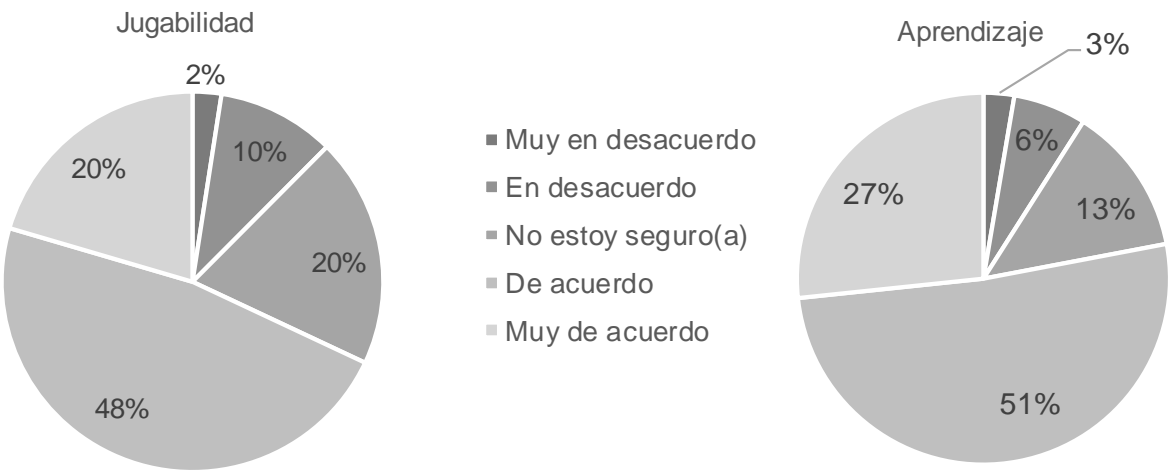

Fig. 8: Respuestas agregadas para las preguntas sobre jugabilidad y aprendizaje

\section{DISCUSIÓN}

Al analizar las respuestas recibidas, es posible determinar que, en el caso los aspectos relacionados a la jugabilidad, el $68 \%$ de los encuestados estuvieron en promedio de acuerdo o muy de acuerdo con los aspectos analizados, frente a un $12.4 \%$ que estuvieron en desacuerdo o muy en desacuerdo. El aspecto con menor tasa de opiniones favorables fue el de la personalización del avatar. Esto quizá se debió, como se muestra en la figura 2, a que las opciones de personalización se limitan a la elección de género y corte de cabello, más la elección de colores del tono de piel, el cabello, y algunas partes del vestuario. Por otra parte, el aspecto con mayor tasa de opiniones desfavorables fue el de la presentación del juego como una aplicación móvil. Estas respuestas dan pie a pensar en la posibilidad de brindar diferentes opciones de plataforma, por lo menos una de computador para aquellos que así lo prefieran.

En el caso los aspectos relacionados a la efectividad en el aprendizaje, el $78 \%$ de los encuestados estuvieron de acuerdo o muy de acuerdo con los aspectos analizados, frente a un $9 \%$ que estuvieron en desacuerdo o muy en desacuerdo. En otras palabras, los estudiantes valoraron más este aspecto del juego, que las características de jugabilidad como tal. Así por ejemplo, el $85.3 \%$ estuvo de acuerdo o muy de acuerdo con la utilidad del juego para comprender la importancia de los hábitos de estudio (pregunta 8), frente a un 84\% relacionado con la comprensión de los hábitos de vida saludable (pregunta 9 ) y un $73.3 \%$ relacionado con la comprensión de los conceptos académicos considerados (pregunta 10). En el caso de dichos hábitos, tanto de estudio como de vida saludable, es importante recordar que son varias las investigaciones que afirman que son unos de los factores más relevantes para lograr una adaptación exitosa (Abe et al., 1998; Credé y Kuncel, 2008; Parker, Yu, 2011; Julia y Veni, 2012). Incluso, en algunas se ahonda en el análisis de estrategias para la apropiación de ellos (Cardona et al. 2001; Mattanah et al.,2010; Credé y Niehorster, 2012), pero basadas en mecanismos más tradicionales como es el caso de las consejerías o las tutorías por pares.

\section{CONCLUSIONES}

Considerando que la adaptación a la vida universitaria es un proceso complejo y en muchas ocasiones incluso traumático para muchos estudiantes, se hace necesario que las universidades brinden mecanismos de inducción para facilitarlo. Sin embargo, más que simplemente brindar información "teórica" al respecto, se requiere el uso de didácticas que faciliten la interiorización de múltiples aspectos que son nuevos y relevantes para los recién admitidos. Este trabajo apunta precisamente a ello, proponiendo el uso de un videojuego móvil. A partir de los resultados obtenidos en la validación realizada con 75 estudiantes de la Universidad Nacional de Colombia Sede Medellín, se comprobó el potencial de la aproximación propuesta en términos de la percepción de diversos elementos tanto de jugabilidad como de aprendizaje. En particular, se puede concluir, no solo que la aproximación presentada resulta atractiva por su formato, sino que su filosofía de brindar una experiencia inmersiva y a la vez lúdica, permite la apropiación eficaz de los cuatro aspectos considerados: la comprensión de nuevos conceptos académicos, los buenos hábitos de estudio, los hábitos de vida saludable, y el reconocimiento del campus.

\section{REFERENCIAS}

Abe, J., D.M.D. Talbot, R. Gellhoed y R.J. Geelhoed. Effects of a Peer Program on International Student Adjustment, J. Coll. Stud. Dev. 39(6), 539-547 (1998).

Annetta, L.A. The 'I's' Have It: A Framework for Serious Educational Game Design, doi:10.1037/a0018985, Rev. Gen. Psychol. 14(2), 105-112 (2010).

Arjanggi, R. y L.P.S. Kusumaningsih. College Adjustment of First Year Students: The Role of Social Anxiety, doi:10.5539/ies.v6n2p172, J. Educ. Heal. Community Psychol. 5(1), 30-39 (2016). 
Bean, J. Dropouts and Turnover: The Synthesis and Test of a Casual Model of Student Attrition. Res. High. Educ. 12, 155187 (1980).

Cabrera, L., J.T. Bethencourt, P. Álvarez y M. González. The Problem of University Dropout, Rev. Electrónica Investig. y Evaluación Educ. 12(2), 171-203 (2006).

Cardona, B.L., M.E. Ramírez y M.B. Tamayo. El Estudiante Recién Llegado en el Escenario Universitario, Rev. Lasallista Investig. 8(2), 76-88 (2011).

Castaño, E., S. Gallón, K. Gómez y J. Vásquez. Análisis de los Factores Asociados a la Deserción Estudiantil en la Educación Superior: Un Estudio de Caso, doi:10.1177/146642405707701004, Rev. Educ. 345, 255-280 (2008).

Corominas, E. La Transición de los Estudios Universitarios. Abandono o Cambio en el Primer Año de Universidad, doi:10.1088/0268-1242/16/4/306, Rev. Investig. Educ. 19(1), 127-151 (2001).

Credé, M. y N.R. Kuncel. Study Habits, Skills, and Attitudes, doi:10.1111/j.1745-6924.2008.00089.x, Prespectives Psychol. Sci. 3(6), 425-454 (2008).

Credé, M. y S. Niehorster. Adjustment to College as Measured by the Student Adaptation to College Questionnaire: A Quantitative Review of its Structure and Relationships with Correlates and Consequences, doi:10.1007/s10648-011-91845, Educ. Psychol. Rev. 24(1), 133-165 (2012).

Díaz, C.J. Modelo Conceptual para la Deserción Estudiantil Universitaria Chilena, doi:10.4067/S071807052008000200004, Estud. Pedagógicos 34(2), 65-86 (2008).

Díaz, C.J. Factores de Deserción Estudiantil en Ingeniería: Una Aplicación de Modelos de Duración, doi:10.1612/inf.tecnol.4095it.08, Inf. Tecnol. 20(5), 129-145 (2009).

Etxeberria, P., E. Alberdi, I. Eguia y M.J. García. Análisis del Rendimiento Académico en Relación al Perfil de Ingreso del Alumnado e Identificación de Carencias Formativas en Materias Básicas de dos Grados de Ingeniería, doi:10.4067/S071850062017000400007, Form. Univ. 10(4), 67-74 (2017).

Figuera, P., I. Dorio y A. Forner. Las competencias académicas previas y el apoyo familiar en la transición a la universidad. Rev. Investig. Educ. 21, 349-369 (2003).

Gallegos, J.A., N.A. Campos, K.A. Canales y E.N. González. Factores Determinantes en la Deserción Universitaria. Caso Facultad de Ciencias Económicas y Administrativas de la Universidad Católica de la Santísima Concepción (Chile), doi:10.4067/S0718-50062018000300011, Form. Univ. 11(3), 11-18 (2018).

Julia, M. y B. Veni. An Analysis of the Factors Affecting Students' Adjustment at a University in Zimbabwe, doi:10.5539/ies.v5n6p244, Int. Educ. Stud. 5(6), 244-250 (2012).

Mattanah, J.F., J. Ayers y otros 4 autores. A Social Support Intervention to Ease the College Transition: Exploring Main Effects and Moderators, doi:10.1353/csd.0.0116, J. Coll. Stud. Dev. 51(1), 93-108 (2010).

Miranda, M.A. y J. Guzmán. Análisis de la Deserción de Estudiantes Universitarios Usando Técnicas de Minería de Datos, doi:10.4067/S0718-50062017000300007, Form. Univ. 10(3), 61-68 (2017).

Munizaga, F.R., M.B. Cifuentes y A.J. Beltrán. Retención y Abandono Estudiantil en la Educación Superior Universitaria en América Latina y el Caribe: Una Revisión Sistemática, doi:10.14507/epaa.26.3348, Educ. Policy Anal. Arch. 26(1), 136 (2018).

Pancer, S.M., B. Hunsberger, M.W. Pratt y S. Alisat. Cognitive Complexity of Expectations and Adjustment to University in the First Year, doi:10.1177/0743558400151003, J. Adolesc. Res. 15(1), 38-57 (2000).

Parker, J.D.A., L.J. Summerfeldt, M.J. Hogan y S.A. Majeski. Emotional Intelligence and Academic Success: Examining the Transition from High School to University, doi:10.1016/S0191-8869(03)00076-X, Pers. Individ. Dif. 36(1), 163-172 (2004).

Spady, W. Dropouts from higher education: Toward an empirical model. Interchange 2, 38-62 (1971).

Taylor, M.A. y D.A. Pastor. A Confirmatory Factor Analysis of the Student Adaptation to College Questionnaire, doi:10.1177/0013164406299125, Educ. Psychol. Meas. 67(6), 1002-1018 (2007).

Tinto, V. Dropout from Higher Education: A Theoretical Synthesis of Recent Research. Rev. Educ. Res. 45, 89-125 (1975).

Vendrell, E. y C. Sánchez. Interactive 3D Visualization of a Large University Campus over the Web. Int. J. Comput, Inf. Syst. Ind. Manag. Appl. 3, 514-521 (2011).

Wang, A., L. Chen, B. Zhao e Y. Xu. First-year Students' Psychological and Behavior Adaptation to College: The Role of Coping Strategies and Social Support, US-China Educ. Rev. 3(5), 51-57 (2006).

Wilson, B. Problems of University Adjustment Experienced by Undergraduates in a Developing Country, High. Educ. 13(1), 1-22 (1984).

Wintre, M.G., G.M. Knoll y otros 5 autores. The Transition to University: The Student-University Match (SUM) Questionnaire, doi:10.1177/0743558408325972, J. Adolesc. Res. 23(6), 745-769 (2008).

Yu, D.D. How Much do Study Habits, Skills, and Attitudes Affect Student Performance in Introductory College Accounting Courses?, doi:10.3168/jds.2010-3644, New Horizons Educ. 59(3), 1-15 (2011). 